\title{
Communication with cancer survivors: Disparities by education level but not race in a majority-minority community
}

Marci Chock ( $\square$ mchock4@hawaii.edu )

University of Hawai'i System

Sharon Tamashiro

Queen's Medical Center

Kristy Fujinaga

Queen's Medical Center

Ryon Nakasone

University of Hawai'i System

Jared Acoba

University of Hawai'i Cancer Center

\section{Research Article}

Keywords: Patient-provider communication (PPC), quality of care, cancer survivors, survivorship, education level

Posted Date: November 10th, 2021

DOI: https://doi.org/10.21203/rs.3.rs-1054249/v1

License: (c) (i) This work is licensed under a Creative Commons Attribution 4.0 International License.

Read Full License 
Communication with cancer survivors: Disparities by education level but not race in a majority-minority community

Author Information:

Marci Chock ${ }^{1}$, Sharon Tamashiro ${ }^{2}$, Kristy Fujinaga ${ }^{3}$, Ryon Nakasone ${ }^{4}$, Jared D. Acoba ${ }^{5}$

Author Affiliations:

${ }^{1}$ University of Hawaii Internal Medicine Residency Program, Honolulu, HI, USA ${ }^{2}$ The Queen's Medical Center, Honolulu, HI, USA ${ }^{3}$ The Queen's Medical Center, Honolulu, HI, USA ${ }^{4}$ University of Hawaii Internal Medicine

Residency Program, Honolulu, HI, USA ${ }^{5}$ University of Hawaii Cancer Center, University of Hawaii Internal

Medicine Residency Program, The Queen’s Medical Center, Honolulu, HI, USA

Corresponding Author:

Marci Chock, mchock4@hawaii.edu 


\begin{abstract}
Purpose

Patient-provider communication (PPC) is an important component of optimal patient care. Many factors influence the quality of PPC among cancer patients, however, there are conflicting data on the impact of patient race and education level on PPC. We sought to assess the effect of race and education level on PPC among a multiracial cohort of cancer survivors.
\end{abstract}

\title{
Methods
}

We conducted a survey of 360 cancer survivors. Data were collected on age, race, income, and education level. The survey assessed satisfaction with PPC and follow-up with cancer physician. Questions were answered on a 5-point response scale. We evaluated PPC using nonparametric analyses and built logistic regression models for satisfaction with follow-up care.

\section{Results}

Patients with a high school or lower education consistently rated questions of PPC more poorly than those who attained a higher level of education. No significant associations were detected between PPC and race. Results for perceived quality of care showed a similar disparity by education level. High school educated patients reported significantly lower satisfaction with quality of follow-up care compared with patients who attained Graduate level education (OR $3.50,95 \%$ CI 1.37-8.94).

\section{Conclusion}

Our study demonstrated that education level, but not race, is associated with perception of communication and satisfaction with follow-up care. Patients who attained higher levels of education had higher PPC ratings and satisfaction with follow-up care. Our findings identify an opportunity for improvement in PPC through individualizing the delivery of health-related information with the goal of achieving optimal care of long-term cancer survivors.

\section{Keywords}

Patient-provider communication (PPC), quality of care, cancer survivors, survivorship, education level 


\section{Declarations}

\section{Conflicts of interest/Competing interests}

The authors have no relevant financial or non-financial interests to disclose.

\section{Availability of data and material}

Not applicable.

\section{Code availability}

Not applicable.

\section{Authors' contributions}

Acoba and Tamashiro contributed to the study conception and design. Material preparation, data collection and

analysis were performed by Tamashiro, Chock and Acoba. The first draft of the manuscript was written by Marci

Chock and all authors commented on previous versions of the manuscript. All authors read and approved the final manuscript.

Additional declarations for articles in life science journals that report the results of studies involving humans and/or animals

Not applicable.

\section{Ethics approval}

IRB approval was granted by the Queen's Medical Center, RA-2018-038.

\section{Consent to participate}

Not applicable.

\section{Consent for publication}

Not applicable. 


\section{Introduction}

The quality of patient-provider communication (PPC) plays a significant role in healthcare through its impact on patient satisfaction, quality of life, treatment compliance, and clinical outcomes. [1-4]. Several studies have demonstrated the influence of cancer patients' socioeconomic status on the perceived quality of PPC [2, 5, 6-7]. In particular, race and education level have been shown to impact patients' perception of communication.

Numerous studies have shown that minority patients rate PPC inferior to white patients. White-Means et al (2017) [8] found that non-Hispanic black and Hispanic breast cancer patients rated their communication with physicians lower than non-Hispanic white patients. Similarly, Asian and Pacific Islander cancer survivors also noted inferior physician communication [7] to white patients. Interestingly, other studies have found no difference in communication [9], and even demonstrated that blacks and Hispanics rate communication better than whites [6]. The association between race and PPC in a multiracial population remains an important topic to further explore.

Perceived suboptimal PPC has also been associated with lower education level. A study of Belgian cancer patients by Aelbrecht et al [9] demonstrated negative patient-physician interactions among individuals lacking language proficiency and those with lower educational attainment. Furthermore, McFarland et al [5] analyzed Hospital Consumer Assessment of Healthcare Providers and Systems (HCAHPS) scores and found that college education was the strongest predictor of higher satisfaction with PPC. However, a previous report by Chawla et al [6] analyzed the Medical Expenditure Panel Survey Experiences with Cancer survey and did not find a clear link between education level and PPC. The role of education in PPC is of particular interest among cancer survivors, for whom effective communication is essential to coordinate patient-centered survivorship care [10].

After completing therapy, cancer survivors face a variety of challenges, including the potential for delayed treatment

effects and the prospect of recurrence or a new primary malignancy [6]. We sought to determine the impact of race and education level on patient satisfaction with PPC among a multiracial cohort of cancer survivors.

\section{Methods}

\section{Data source and sample population}


This study was conducted at the Queen's Medical Center (QMC), the largest hospital system in the state of Hawaii (IRB approval, RA-2018-038). QMC's Cancer Program is accredited by the Commission on Cancer (COC), and has an established survivorship program. All patients who complete cancer treatment with curative intent meet with QMC's survivorship nurse and are given a survivorship care plan. Cancer survivors who completed their treatment between January 1, 2014 and June 30, 2018 were eligible to participate. Responses were collected from November 2018 through January 2019. Participants needed to be literate in English. Approval for the study was obtained from the institutional review board at QMC.

\section{Sociodemographic and clinical factors}

We examined sociodemographic variables including age, sex, race, education, and income. Age was categorized as younger than 60 years, 60-69 years, and 70 years and older. Categories for income included: prefer not to say, $<\$ 30,000$ per year, $\$ 30,000-89,999$ per year, and $\$ 90,000$ and more per year. Study participants self-identified their race and were grouped as either white, Native Hawaiian or Pacific Islander (NH/PI), or Asian. Survivors also selfreported their education level and were categorized as high school or less, some college education but not a bachelor's degree, bachelor's degree, or a master's or doctorate degree.

Cancer information was similarly self-reported and included the following: cancer type (breast, lung, gynecologic, gastrointestinal, head and neck, genitourinary, or other), year of diagnosis (prior to 2016 or 2016-2018), and cancer treatment (yes or no for surgery, radiation, and chemotherapy).

\section{Measures}

\section{Patient-provider communication}

Study participants answered questions on PPC adapted from a prior study of cancer survivors (Palmer et al, 2014): How often did your cancer-related follow-up physician (1) listen carefully to you, (2) explain things in a way you could understand, (3) show respect for what you had to say, (4) encourage you to ask all the cancer-related questions you had, (5) make sure you understood all of the information he or she gave you, (6) spend enough time with you, (7) give you as much cancer-related information as you wanted, and (8) involve you in decisions about your medical care 
as much as you wanted. Responses were assessed on a 5-point response scale, 1 (Never) to 5 (Always). We dichotomized responses because of the negatively skewed data (1-3 vs 4 or 5).

\section{Perceived quality of care}

Patients were asked to rate the quality of their follow-up with their cancer physician. Responses were assessed on a 5point response scale (Poor to Excellent). We dichotomized responses because of the negatively skewed data (Poor to Very Good vs Excellent).

\section{Statistical analysis}

Nonparametric descriptive statistics were used to evaluate characteristics of standard demographic data, tabulated by education level. A p value $<0.05$ was considered statistically significant. PPC and overall quality of care was analyzed with scoring endpoints described above. Logistic regression models for satisfaction with follow-up visits were built to obtain odds ratios (OR) and 95\% confidence intervals (CI). Multivariate models were adjusted for age, sex, race, income, education level, and cancer information (stage, year of diagnosis, treatment). Statistical analyses were performed with SPSS version 27.0 (IBM Corporation).

\section{Results}

\section{Sample characteristics}

A total of 1410 long-term cancer survivors were sent the survey between November 2018 through January 2019, with a response rate of 25.5\% (360 patients). Education level was available for $345(95.8 \%)$ cancer survivors. Study population characteristics stratified by education level are listed in Table I. The sample consisted of $15 \%$ high school or less educated patients (High School), 32\% with some college education but not a bachelor's degree (Some College), $32 \%$ with a bachelor's degree (Bachelor's), and 28\% with a master's or doctorate degree (Graduate). Asians comprised $65 \%$ of the study population, followed by whites at $24 \%$, and NH/PI at $12 \%$. A majority of the patients had an income of $\geq \$ 60,000$ and were diagnosed within three years of completing the survey. Significant differences among education groups were noted by race, income, year diagnosed, and whether or not treatment included surgery or radiation therapy. High school educated patients were more likely to have a lower income and respondents with a graduate degree were more likely to be white. 


\section{Education level differences in PPC and quality of care ratings}

Patients with a high school or lower education consistently rated questions of PPC more poorly than those who attained a higher level of education (Table II). There were significant differences by education level when patients were asked the following: How often did your cancer physician: listen carefully to you $(\mathrm{p}=0.024)$, explain things well $(\mathrm{p}<0.0005)$, encourage questions ( $\mathrm{p}=0.043)$, and involve you in decision making $(\mathrm{p}=0.002)$.

Overall satisfaction with follow-up care was also significantly associated with education level $(\mathrm{p}=0.028)$. High school educated patients reported significantly lower satisfaction with quality of follow-up care compared with patients who attained Graduate level education (OR 3.06, 95\% CI 1.45-6.46) (Table III). This disparity persisted after adjustment for race, income, and cancer information (vs Graduate, OR 3.50, 95\% CI 1.37-8.94). There were no significant income disparities or difference by cancer type or treatment in PPC or quality of care ratings.

\section{Race and PPC}

There were no significant differences among race and rating of PPC (Table IV). Quality of care was also evaluated similarly among white, Asian, and NH/PI patients.

\section{Discussion}

Our study demonstrated that patients with a lower education level rated their communication with their cancer physician less favorably as compared to patients who attended college. However, our study did not show an association between race and PPC. In contrast to previous studies [7, 11], we did not find a racial disparity in PPC. We hypothesize two reasons that could account for this result. First, Hawaii is a minority-majority state, and minority cancer patients receive care at the same clinical centers as whites. Gaskin et al [12] demonstrated that minorities have similar outcomes as whites when treated at the same center. Second, racial concordance between patients and providers $[13,14]$ has been shown to improve PPC. The majority of the oncology providers caring for patients who participated in this study are racial minorities.

To our knowledge, this is the first study to demonstrate that education level is associated with PPC specifically among cancer survivors. Other studies of cancer patients on active treatment have demonstrated similar findings. Cancer patients with a higher education level have been shown to be more willing to share their emotional concerns [15] and 
want a more active role in their treatment decisions [16], implying improved communication with their oncologist. Moreover, Hagerty et al [17] found that cancer patients with a lower education level were more likely to misunderstand their illness.

Effective communication between patient and provider can improve patient care outcomes [5]. Arora et al [18] showed that cancer survivors' perception of overall care is closely associated with their assessment of the quality of their communication with their physician. Identification of specific interventions to improve communication among patients with a lower education level such as the "teach back" technique and integration of various types of media including pictures, video, and stories $[19,20]$ can be assessed with the ultimate goal of improving clinical outcomes. However, methods of communication may be most effective if they are individualized to each patient, as patients vary in their communication preferences and desire to play an active role in their care [21].

This study has a number of strengths including the surveillance of a multiracial population of cancer survivors with a high proportion of NH/PI. There were also a broad variety of cancer types represented by the cohort reflecting a realworld cancer center. There are also several limitations of this study. First, the cohort includes a limited number of patients, and while it was a multiracial population, not all races (such as African American) were represented. Second, our respondents had a relatively high income and the majority were college graduates; this may not be generalizable to all cancer centers. Third, although the survey questions were based on previously published study of PPC among cancer patients, we did not use a validated tool to assess communication.

\section{Conclusion}

Our study demonstrated that the education level of cancer survivors is associated with their perception of communication and satisfaction with follow-up care. While race has been shown in previous studies to impact PPC, this was not demonstrated in our multiracial patient population. One may extrapolate that greater awareness of the need to cater delivery of information to different education levels may improve patient satisfaction and overall health outcomes. Future research to identify methods of communication that are most satisfactory across a broad spectrum of different education levels could improve the care of cancer survivors. 


\section{References}

1. Li CC, Matthews AK, Dossaji M, \& Fullam F (2017) The relationship of patient-provider communication on quality of life among African-American and white cancer survivors. Journal of health communication, 22(7), 584-592.

2. Hall JA, Roter DL, \& Rand CS (1981) Communication of affect between patient and physician. Journal of health and social behavior, 18-30.

3. Ong LM, Visser MR, Lammes FB, \& De Haes JC (2000) Doctor-patient communication and cancer patients' quality of life and satisfaction. Patient education and counseling, 41(2), 145-156.

4. Cella DF, Orav EJ, Kornblith AB, et al (1991) Socioeconomic status and cancer survival. Journal of Clinical Oncology, 9(8), 1500-1509.

5. McFarland DC, Johnson Shen M, \& Holcombe RF (2017) Predictors of satisfaction with doctor and nurse communication: A national study. Health communication, 32(10), 1217-1224.

6. Chawla N, Blanch-Hartigan D, Virgo KS, et al (2016) Quality of patient-provider communication among cancer survivors: findings from a nationally representative sample. Journal of oncology practice, 12(12), e964-e973.

7. Palmer NR, Kent EE, Forsythe LP, et al (2014) Racial and ethnic disparities in patient-provider communication, quality-of-care ratings, and patient activation among long-term cancer survivors. Journal of Clinical Oncology, 32(36), 4087.

8. White-Means SI \& Osmani AR (2017) Racial and ethnic disparities in patient-provider communication with breast cancer patients: evidence from 2011 MEPS and experiences with cancer supplement. INQUIRY: The Journal of Health Care Organization, Provision, and Financing, 54, 0046958017727104.

9. Aelbrecht K, Hanssens L, Detollenaere J, Willems S, Deveugele M, \& Pype P (2019) Determinants of physician-patient communication: The role of language, education and ethnicity. Patient education and counseling, 102(4), 776-781.

10. Rai A, Han X, Zheng Z, Yabroff KR, \& Jemal A (2018) Determinants and outcomes of satisfaction with healthcare provider communication among cancer survivors. Journal of the National Comprehensive Cancer Network, 16(8), 975-984.

11. Maly RC, Stein JA, Umezawa Y, Leake B, \& Anglin MD (2008) Racial/ethnic differences in breast cancer outcomes among older patients: effects of physician communication and patient empowerment. Health Psychology, 27(6), 728.

12. Gaskin DJ, Spencer CS, Richard P, Anderson GF, Powe NR, \& LaVeist TA (2008) Do hospitals provide lowerquality care to minorities than to whites?. Health affairs, $27(2), 518-527$.

13. Shen MJ, Peterson EB, Costas-Muñiz R, Hernandez MH, Jewell ST, Matsoukas K, \& Bylund CL (2018) The effects of race and racial concordance on patient-physician communication: a systematic review of the literature. Journal of racial and ethnic health disparities, 5(1), 117-140.

14. Saha S \& Beach MC (2020) Impact of physician race on patient decision-making and ratings of physicians: a randomized experiment using video vignettes. Journal of general internal medicine, 1-8.

15. Back A (2006) Patient-physician communication in oncology: what does the evidence show?. Oncology (Williston Park, NY), 20(1), 67-74. 
16. Janz NK, Wren PA, Copeland LA, Lowery JC, Goldfarb SL, \& Wilkins EG (2004) Patient-physician concordance: preferences, perceptions, and factors influencing the breast cancer surgical decision. Journal of clinical oncology, 22(15), 3091-3098.

17. Hagerty RG, Butow PN, Ellis PM, Dimitry S, \& Tattersall MHN (2005) Communicating prognosis in cancer care: a systematic review of the literature. Annals of oncology, 16(7), 1005-1053.

18. Arora NK, Reeve BB, Hays RD, Clauser SB, \& Oakley-Girvan I (2011) Assessment of quality of cancer-related follow-up care from the cancer survivor's perspective. Journal of Clinical Oncology, 29(10), 1280.

19. Davis TC, Williams MV, Marin E, Parker RM, \& Glass J (2002) Health literacy and cancer communication. CA: a cancer journal for clinicians, 52(3), 134-149.

20. Paasche-Orlow MK, Schillinger D, Greene SM, \& Wagner EH (2006) How health care systems can begin to address the challenge of limited literacy. Journal of General Internal Medicine, 21(8), 884-887.

21. Rodin G, Zimmermann C, Mayer C, et al (2009) Clinician-patient communication: evidence-based recommendations to guide practice in cancer. Current Oncology, 16(6), 42. 
Table I: Baseline characteristics stratified by education

Education Level, n (\%)

\begin{tabular}{|c|c|c|c|c|c|}
\hline Characteristic & High School a & Some College ${ }^{b}$ & Bachelor's $^{c}$ & Graduate $^{d}$ & $p$ value \\
\hline Age & & & & & 0.89 \\
\hline$\leq 60$ & $10(20)$ & $23(21)$ & $24(22)$ & $20(27)$ & \\
\hline $60-69$ & $21(40)$ & $46(42)$ & $39(36)$ & $26(35)$ & \\
\hline$\geq 70$ & $21(40)$ & $40(37)$ & $46(42)$ & $29(39)$ & \\
\hline Sex & & & & & 0.23 \\
\hline Male & $31(59)$ & $78(72)$ & $66(60)$ & $51(68)$ & \\
\hline Female & $22(42)$ & $31(28$ & $43(39)$ & $24(32)$ & \\
\hline Race & & & & & $<0.0005$ \\
\hline White & $11(21)$ & $17(16)$ & $21(19)$ & $35(47)$ & \\
\hline Asian & $29(55)$ & $67(62)$ & $78(72)$ & $31(41)$ & \\
\hline $\mathrm{NH} / \mathrm{PI}{ }^{\mathrm{e}}$ & $9(17)$ & $18(17)$ & $8(7)$ & $7(9)$ & \\
\hline Declined/Other & $4(8)$ & $6(6)$ & $2(2)$ & $2(3)$ & \\
\hline Income & & & & & $<0.0005$ \\
\hline$\leq 30,000 / \mathrm{yr}$ & $19(37)$ & $19(18)$ & $4(4)$ & $1(1)$ & \\
\hline $30,000-59,000 / \mathrm{yr}$ & $14(27)$ & $23(21)$ & $19(18)$ & $6(8)$ & \\
\hline $60,000-89,000 / \mathrm{yr}$ & $6(12)$ & $20(19)$ & $25(23)$ & $17(23)$ & \\
\hline$\geq 90,000 / \mathrm{yr}$ & $4(8)$ & $29(27)$ & $43(40)$ & $37(50)$ & \\
\hline Declined & $9(17)$ & $17(16)$ & $17(16)$ & $13(18)$ & \\
\hline Year diagnosed & & & & & 0.011 \\
\hline Prior to 2016 & $19(37)$ & $60(56)$ & $39(36)$ & $39(52)$ & \\
\hline $2016-2018$ & $33(63)$ & $48(44)$ & $69(64)$ & $36(48)$ & \\
\hline Cancer type & & & & & 0.97 \\
\hline Breast & $19(39)$ & $39(36)$ & $42(39)$ & $30(40)$ & \\
\hline
\end{tabular}




\begin{tabular}{|c|c|c|c|c|c|}
\hline Lung & $3(6)$ & $11(10)$ & $5(5)$ & $4(5)$ & \\
\hline Gynecologic & $6(12)$ & $14(13)$ & $9(8)$ & $8(11)$ & \\
\hline Gastrointestinal & $3(6)$ & $8(7)$ & $10(9)$ & $5(7)$ & \\
\hline Head and neck & $4(8)$ & $11(10)$ & $8(8)$ & $8(11)$ & \\
\hline Genitourinary & $7(14)$ & $13(12)$ & $21(20)$ & $13(17)$ & \\
\hline Other & $7(14)$ & $13(12)$ & $12(11)$ & $7(9)$ & \\
\hline Surgery & & & & & 0.035 \\
\hline Yes & $39(77)$ & $95(87)$ & $100(93)$ & $67(89)$ & \\
\hline No & $12(23)$ & $14(13)$ & $8(7)$ & $8(11)$ & \\
\hline Chemotherapy & & & & & 0.88 \\
\hline Yes & $23(44)$ & $50(46)$ & $42(39)$ & $34(45)$ & \\
\hline No & $29(56)$ & $58(53)$ & $65(60)$ & $41(55)$ & \\
\hline Radiation & & & & & 0.027 \\
\hline Yes & $39(75)$ & $63(59)$ & $75(69)$ & $40(53)$ & \\
\hline No & $13(25)$ & $45(41)$ & $33(31)$ & $35(47)$ & \\
\hline
\end{tabular}

${ }^{\text {a }}$ high school or less educated patients; ${ }^{\mathbf{b}}$ some college education but not a bachelor's degree; ' bachelor's degree;

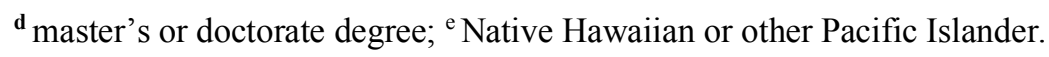


Table II: Satisfaction with communication by highest education level attained

Number of responders answering "Most Often" or "Excellent" (\%)

\section{How often does your cancer} physician...

listen carefully to you

explain things well

respect what you say

encourage questions

ensure understanding

spend enough time

provide information

involve you in decision making

Quality of follow-up care
High School

$45(87)$

$45(85)$

48 (91)

$41(80)$

45 (88)

$45(87)$

$41(82)$

41 (79)

$21(42)$
$100(94)$

102 (96)

102 (96)

94 (90)

98 (93)

95 (90)

93 (88)

98 (93)

$61(59)$
Bachelor

106 (97)

106 (97)

106 (97)

$100(93)$

104 (96)

$31(100)$

99 (92)

102 (94)

65 (61)
$51(69)$

0.024

$75(100) \quad<0.0005$

74 (99)

NS ${ }^{a}$

0.043

NS

NS

NS

0.002

0.028

\footnotetext{
${ }^{a}$ Not significant.
} 
Table III: Models of education differences in satisfaction with follow-up care

\begin{tabular}{lccc} 
& OR & $\mathbf{( 9 5 \%} \mathbf{C I})$ & $\boldsymbol{p}$ value \\
\cline { 2 - 4 } Univariate analysis & -- & & \\
High school & 1.96 & $(0.99-3.88)$ & 0.054 \\
Some college & 2.14 & $(1.08-4.23)$ & 0.029 \\
Bachelor & 3.06 & $(1.45-6.46)$ & 0.003 \\
Graduate & &
\end{tabular}

Multivariate analysis; adjusted for race and income

High school

Some college $\quad 2.5$

$\begin{array}{ll}(1.11-5.76) & 0.027\end{array}$

Bachelor

2.34

$(1.01-5.45)$

0.048

Graduate

3.50

$(1.37-8.94)$

0.009 
Table IV: Satisfaction with communication by race

Number of responders answering "Most Often" or "Excellent" (\%)

How often does your cancer

physician...

listen carefully to you
explain things well
respect what you say
encourage questions
ensure understanding
spend enough time
provide information
involve you in decision making

Quality of follow-up care

${ }^{a}$ Not significant.

\begin{tabular}{llll}
\hline White & Asian & NH/PI & $p$ value
\end{tabular}

$79(93) \quad 199(95) \quad 40(93) \quad \mathrm{NS}^{\mathrm{a}}$

$82(97) \quad 199(95) \quad 40(93) \quad$ NS

$82(97) \quad 199(95) \quad 42(98) \quad$ NS

$79(95) \quad 185(89) \quad 37(88) \quad$ NS

$79(95) \quad 195(94) \quad 39(93) \quad$ NS

$75(89) \quad 189(90) \quad 39(93) \quad$ NS

$77(92) \quad 181(87) \quad 39(93) \quad$ NS

$79(95) \quad 190(91) \quad 37(88) \quad$ NS

$55(66) \quad 113(55) \quad 26(62) \quad$ NS 